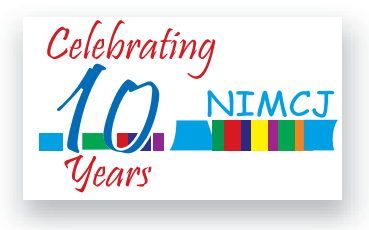

1 Prof. Mahmuda Hassan Prof. of paediatrics Ad-din Women's Medical College

2 Dr. Hamidur Rahman Prof. and head

3 Prof. B H Nazma Yasmeen Prof. and head Dept. of Paediatrics Nothern International Medical College, Dhaka

4

Dr. Afsana Mukti Assistant Professor

5

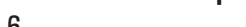

6 Dr. Masuma Khan Assistant Professor

7

Marium Begum

8 Dr. Sudipta Roy Assistant Professor

9 Prof. Taslim Uddin Ahmed

${ }^{10}$ Prof. Ashraf Uddin Ahmed

${ }^{11}$ Dr. Sadia Tabassum Asstt. Registrar of Paediatrics Shahid Sarwardi Medical

${ }^{12}$ Prof. A R M Luthful Kabir

$2,4,6,8,9,10,12$

Dept. of Paediatrics

Ad-din Women's Medical College 5,7

Associate Professor

Basundhara Ad-din Medical

College, Dhaka

Correspondence

Prof. M Hasan

Prof. of paediatrics

Ad-din Women's Medical College

e-mail: mahmudahasan@yahoo.com

\title{
Thiamine deficiency - Beriberi - A forgotten disease
}

\author{
M Hasan ${ }^{1}$, H Rahman ${ }^{2}$, B H N Yasmeen ${ }^{3}$, A Mukti ${ }^{4}$, H Haque ${ }^{5}$, M Khan ${ }^{6}$, M Begum ${ }^{7}$, \\ S Roy ${ }^{8}$, T U Ahmed ${ }^{9}$, AU Ahmed ${ }^{10}$, S Tabassum ${ }^{11}$, A R M L Kabir ${ }^{12}$
}

\section{Abstract}

Thiamine (vitamin B1) was the first B vitamin which has been identified. It serves as a cofactor for several enzymes involved in energy metabolism. The thiamine-dependent enzymes are important for the biosynthesis of neurotransmitters and for the production of reducing substances used in oxidant stress defenses, as well as for the synthesis of pentose used as nucleic acid precursors. Thiamine also plays a central role in cerebral metabolism.

There are 2 major manifestations of thiamine deficiency(TD):

cardiovascular disease (wet beriberi) and nervous system disease (dry beriberi and Wernicke-Korsakoff syndrome).

In wet beriberi, cardiomyopathy with edema and lactic acidosis and in dry beriberi, peripheral neuropathy occurs. Manifestations of Wernicke-Korsakoff syndrome, consist of nystagmus, ophthalmoplegia and ataxia evolving into confusion, retrograde amnesia, cognitive impairment and confabulation.

Thiamine deficiency is now very rare in developed countries, but still common in South East Asia specially in developing countries like Bangladesh. It is an important public health problem with potentially fatal consequences. Now a days highly polished rice (Minicut rice) is a popular staple food and other primary dietary sources of thiamine are in short supply.

In wet beriberi myocardial disease is prominent which causes a high cardiac output with peripheral vasodilation and warm extremities. Before heart failure, tachycardia, a wide pulse pressure, sweating, warm skin and lactic acidosis develop leading to salt and water retention by the kidneys. The resulting fluid overload leads to edema of the dependent extremities. If it is left untreated the severity of potential outcome will be increased even up to death. Therefore, makes it essential for physician, cardiologists and Paediatrician to have an understanding of this condition and its optimal treatment.

Patients on a strict thiamine-deficient diet display a state of severe depletion within 18 days. The most common cause of thiamine deficiency in affluent countries is either alcoholism or malnutrition in nonalcoholic patients especially in children. Treatment by thiamine supplementation is beneficial for diagnostic and therapeutic purposes.

Keywords : Thiamine, Vitamin B1, Beriberi, Cardiomyopathy, Neuropathy, Wernicke-Korsakoff syndrome,

\section{Introduction}

Thiamine (also known as vitamin B1 or aneurin) was the first $B$ vitamin discovered. Beriberi is a 'forgotten disease' that remains a public health issue in Southeast Asia including Bangladesh, despite near eradication elsewhere. ${ }^{1-4}$

It remains as a problem in Southeast Asia, because of non-parboiled, unfortified white rice is the dietary staple here. Although rice contains thiamine, which is found only in the outer husk and bran, the vast majority of which is removed during the milling process. $^{5}$ In most riceconsuming cultures, polished white rice is preferred for several reasons: organoleptic qualities, white rice is a symbol and because removal of the lipid-rich outer bran increases shelf-life. $^{5}$

Thiamine requirements are closely related to carbohydrate intake. In rice diets, $75 \%$ or more of the energy is provided by carbohydrate. Thiamine needs have usually been expressed as mg per $1000 \mathrm{kcal}$ energy from carbohydrate intake. However, the error involved in relating the thiamine content of a diet to the total energy content of the diet rather than to the energy derived from carbohydrate. Thiamine 
deficiency occurs where the diet consists mainly of milled white cereals, including polished rice, and wheat flour, all very poor sources of thiamine. Thiamine deficiency can develop within 2-3 months of a deficient intake. It can cause disability and death. Thiamine deficiency in refugees has been seen in Thailand at the beginning of the 1980's and in the 1990's, in Guinea (1990), Djibouti (1993) and in Nepal (1993-1995). ${ }^{6-8}$

Beriberi is caused by thiamine (vitamin B1) deficiency and is most serious in infants due to their rapid growth and development that occurs during this time and the relatively high thiamine needs compared to body size. Breast milk thiamine concentrations reflect maternal dietary thiamine intake. ${ }^{9}$ As such, poor maternal thiamine status during pregnancy and lactation puts infants at risk of developing beriberi which can lead to death in hours of clinical presentation if not recognized or left untreated. ${ }^{10}$ While infantile beriberi is the most serious outcome of thiamine deficiency, marginal thiamine status in the wider population causes fatigue, apathy, anorexia and dizziness, and with these the potential for decreased school performance and/or economic output.

\section{Thiamine Deficiency States}

Primary thiamine deficiency is caused by inadequate intake of thiamine, most commonly in under developed countries. The great outbreaks of thiamine deficiency in Southeast Asia at the beginning of the 20th century followed the large-scale production of milled rice and its extensive distribution.

Secondary thiamine deficiency is caused by increased requirement, as in hyperthyroidism, pregnancy, lactation and fever. It is also associated with impaired absorption, as in prolonged diarrheas and impaired utilization, as in severe liver disease.

There are 2 major manifestations of thiamine deficiency : cardiovascular disease (wet beriberi) and nervous system disease (dry beriberi and Wernicke-Korsakoff syndrome).

Wet beriberi occurs in thiamine deficiency when myocardial disease is prominent. This causes a high cardiac output with peripheral vasodilation and warm extremities. Before heart failure occurs, tachycardia, a wide pulse pressure, sweating, warm skin, and lactic acidosis develop, leading to salt and water retention by the kidneys. The resulting fluid overload leads to edema of the dependent extremities. A more rapid form of wet beriberi has been termed acute fulminant cardiovascular beriberi with lactic acidosis or Shoshin beriberi, in which vasodilation continues, resulting in shock in a patient with heart failure. ${ }^{11}$

Symptoms of dry beriberi are bilateral and symmetric, predominantly involving the lower extremities and beginning with paresthesia of the toes, burning of the feet (particularly severe at night), muscle cramps in the calves, and pain in the legs. Calf muscle tenderness, difficulty in rising from a squatting position, a decrease in the vibratory sensation in the toes, and plantar dysesthesia are early signs. A diagnosis of mild peripheral neuropathy can be made when ankle jerks are absent. Continued deficiency causes loss of knee jerk, loss of vibratory and position sensation in the toes, atrophy of the calf and thigh muscles and finally foot drop and toe drop. Arms can be affected after leg signs are well established.

Wernicke-Korsakoff syndrome or Wernicke encephalopathy is the thiamine-deficient disease seen most often in the Western hemisphere. It mainly affects alcoholics because of a number of reasons: (a) their diet is usually poor; (b) diets rich in carbohydrates (eg. rice) increase the metabolic demands of thiamine.

In Wernicke-Korsakoff syndrome- manifestations consist of nystagmus, ophthalmoplegia and ataxia evolving into confusion, retrograde amnesia, cognitive impairment and confabulation.

Infantile beriberi usually occurs between the second and fourth month of life who are breastfed by thiamine deficient mothers. The onset of symptoms is often very rapid and the fatality rate is very high. Initially, an infant with thiamine deficiency has a normal appearance with varying degrees of constipation, occasional vomiting, crying, and restlessness. Subsequently, the disease usually presents with cardiac manifestations or can display meningeal irritation accompanied by vomiting and convulsions (the pseudomeningitic form). Heart failure, aphonia, and absent deep tendon reflexes are characteristic of progressive disease. ${ }^{12}$

\section{Early Symptoms}

The earliest symptoms of thiamine deficiency are nonspecific and include fatigue, irritation, poor memory, sleep disturbances, precordial pain, anorexia, abdominal discomfort and constipation. Five stages of the development of a vitamin deficiency were described by Brin in 1964. ${ }^{13}$

First or preliminary stage, inadequate thiamine availability because of faulty diet, malabsorption, or abnormal metabolism leads to a greatly reduced urinary thiamine loss.

Second or biochemical stage, the activity of transketolase is significantly reduced.

Third, or physiologic stage, various general symptoms develop, such as reduced appetite, insomnia, increased irritability and malaise.

Fourth, or clinical stage, a constellation of symptoms classically specific to thiamine-deficiency disease (beriberi) develops, including intermittent claudication, polyneuritis, bradycardia, peripheral edema, cardiac enlargement and ophthalmoplegia.

Fifth, or anatomical stage, histopathological changes because of 
cellular structural damage are seen, such as cardiac hypertrophy, degeneration of the granular layer of the cerebellum, and swelling of the brain microglia.

It is noteworthy that the first 3 stages do not involve specific signs of deficiency.

\section{Factors affecting thiamine requirements}

The following factors have been found to influence thiamine requirements (WHO, 1967):

\section{Composition of the diet}

Experimental studies with adults and infants have indicated that thiamine requirement is related to the dietary ratio of carbohydrates to fat. However, a major increase in the proportion of fat in the diet was found to have a comparatively small effect on thiamine requirements. Thus, diets exceedingly high in carbohydrates appear to increase the requirement for thiamine. Proteins in the diet play an intermediate role between fats and carbohydrates with regard to their metabolic influence on thiamine requirements.

Carbohydrate and protein calories more closely represent the total calorie intake of people in developing countries. Although this fact may result in an increase in the thiamine required per 1000 calories.

\section{Climate}

Climatic changes involving cold or hot temperatures increase or decrease energy expenditure respectively and therefore increase or decrease requirements.

\section{Body weight}

The thiamine requirement will vary with body weight to the extent that energy requirements are related to body weight

\section{Physical activity}

The increase in energy requirements which accompanies increased physical activity leads to a corresponding increase in the absolute requirement of thiamine with no alteration in the thiamine to calorie ratio.

\section{Age}

Minimal requirements for dietary thiamine may increase with age, particularly for active individuals.

\section{Pathological conditions}

Conditions such as infections, gastrointestinal disease, thyroid disorders, trauma or chronic over consumption of alcohol significantly modify the requirement for thiamine in relation to calories. Thiamine nutriture is said to be sufficient provided the recommended thiamine to calorie ratio is maintained.

\section{Anti-thiamine factors}

Thiamine in foods can be destroyed by anti-thiamine compounds that occur naturally in some food. The condition was brought on by a thiamine-degrading enzyme (thiaminase) present in fish gut tissue.

Heat-stable thiamine antagonists occur in several plants; ferns, tea, betel nut. They include polyphenols; these and related compounds are found in blue berries, red currants, red beets, brusselsprouts, red cabbage, betel nuts, coffee and tea. ${ }^{14}$

Some bacteria (e.g. Bacillus thiamineolyticus) are also capable of destroying thiamine. It has been reported that $3 \%$ of Japanese show a thiamine deficiency due to this cause. tea drinking and chewing of fermented tea leaves; tannins being the major component having anti-thiamine activity, ascorbic acid (vitamin C) protect the modification of thiamine by tea extract.

The following recommendations were made to decrease the influence of anti-thiamine factors in reducing thiamine absorption

- delay the consumption of tea or other tannin-containing products after a meal

- consume foods high in ascorbic acid along with the meals

- heat products containing thiaminase before consumption ${ }^{15}$

\section{Pathological Findings of Thiamine Deficiency}

The most advanced neural changes occur in the peripheral nerves, particularly of the legs. The distal segments are characteristically affected earliest and most severely. Degeneration of the medullary sheath can occur in all tracts of the spinal cord, especially in the posterior columns and in the anterior and posterior nerve roots. The heart is dilated and enlarged and muscle fibers are swollen, fragmented and vacuolized, with interstitial spaces dilated by fluid. Vasodilation can result in some edema before high-output heart failure occurs. The pathology of the brain in thiamine deficiency consists of bilaterally symmetrical midline hemorrhagic and/or necrotic lesions in selective brain regions, mainly the mammillary bodies, thalamus (medial dorsal, anterior medial, and pulvinar), periaqueductal region and floor of the fourth ventricle, hypothalamus and cerebellar vermis. ${ }^{16,17}$

\section{Treatment of Thiamine Deficiency}

For mild polyneuropathy, 10 to $20 \mathrm{mg} /$ day of thiamine is given individed doses for 2 weeks followed by a nutritious diet.

The dosage is 20 to $30 \mathrm{mg} /$ day for moderate or advanced neuropathy and should be continued for several weeks after the symptoms disappear. 
The edema and congestion of Shoshin beriberir respond to 100 $\mathrm{mg} /$ day of thiamine intravenously, which should be continued for several days.

Heart failure due to beriberi responds poorly to digitalis or diuretics.

For Wernicke-Korsakoff syndrome, thiamine 50 to $100 \mathrm{mg}$ intramuscularly or intravenously twice a day must usually be given for several days, followed by 10 to $20 \mathrm{mg}$ daily until a therapeutic response is obtained.

Anaphylactic reactions to intravenous thiamine unrelated to the dose are rare. This regimen should be followed indefinitely by a nutritious diet supplying 1 to 2 times the recommended dailyallowances. Magnesium, a cofactor for transketolase, should be given as magnesium sulfate with thiamine to correct thiamine resistance and the frequently accompanying hypomagnesemia. Recovery from neurologic deficits is often incomplete in beriberi.

\section{Conclusion}

Clinicians should be vigilant and consider the possibility of thiamine deficiency in high-risk patients. If the diagnosis is questionable, it is recommended to treat the patient with thiamine any how, given that it is a safe and inexpensive therapeutic agent, which can prevent irreversible damage. Preventive strategies might be effective on the national level and countries should consider this by supplementing staple foods with thiamine.

\section{References}

1. Lee HS, Lee SA, Shin HS, Choi HM, Kim SJ, Kim HK, et al. A case of cardiac beriberi: a forgotten butmemorable disease. Korean Circ J. 2013; 43: 569572 .

2. Lonsdale D. A review of the biochemistry, metabolism and clinical benefits of thiamin(e) and its derivatives.e CAM Evidence-Based Complement Altern Med. 2006; 3: 49-59.

3. Soukaloun D, Lee SJ, Chamberlain K, Taylor AM, Mayxay M, Sisouk K, et al. Erythrocyte transketolaseactivity, markers of cardiac dysfunction and the diagnosis of infantile beriberi. PLoSNegl Trop Dis.2011; 5: e971
4. Coats D, Shelton-Dodge K, Ou K, Khun V, Seab S, Sok K, et al. Thiamine deficiency in Cambodianinfants with and without beriberi. J Pediatr. 2012; 161: 843-847.

5. Vanier NL, Paraginski RT, Berrios JDJ, Oliveira LDC, Elias MC. Thiamine content and technological quality properties of parboiled rice treated with sodium bisulfite: benefits and food safety risk. J Food Compos Anal. 2015; 41: $98-103$

6. World Health Organization. Thiamine Deficiency and Its Prevention and Control in Major Emergencies. Geneva, Switzerland:Department of Nutrition for Health and Development, World Health Organization; 1999.

7. Thanangkul $O$, Whitaker JA, Fort EG. Malnutrition in northern Thailand. Am J ClinNutr. 1966;18:379-389.

8. TangCM, RolfeM,Wells JC, Cham K. Outbreak of beri-beri in The Gambia. Lancet. 1989;2:206-207.

9. Allen LH. B vitamins in breast milk: relative importance of maternal status and intake, and effects oninfant status and function. AdvNutr. 2012; 3: 362- 69.

10. Ortega RM, MartõÂnez RM, AndreÂs P, MarõÂn-Arias L, LoÂpez-Sobaler AM. Thiamin status during the thirdtrimester of pregnancy and its influence on thiamin concentrations in transition and mature breast milk.Br J Nutr. 2004; 92: $129-35$.

11.Yamasaki H, Tada H, Kawano S, Aonuma K. Reversible pulmonary hypertension, lactic acidosis, and rapidly evolving multiple organ failure as manifestations of shoshin beriberi. Circ].2010;74:1983-1985.

12. Vasconcelos MM, Silva KP, Vidal G, Silva AF, Domingues RC, Berditchevsky CR. Early diagnosis of pediatric Wernicke's encephalopathy. Pediatr Neurol. 1999;20:289-294.

13. BrinM. Erythrocyte as a biopsy tissue for functional evaluation of thiamine adequacy. JAMA. 1964;187:762-766.

14. Hilker DM, Somogyi JC. Antithiamines of plant origin: their chemical nature and mode of action. [Review]. Annals of the New York Academy of Sciences, 1982, health and disease. 378:137-144.

15. Vimokesant $\mathrm{S}$ et al. Beriberi caused by antithiamine factors in food and its prevention. [Review] Annals of the New YorkAcademy of Sciences, 1982, 378: 123-136.

16. Victor M, Adams RD, Collins GH. The Wernicke-Korsakoff syndrome. A clinical and pathological study of 245 patients,82 with post-mortem examinations. Contemp Neurol Ser. 1971;7:1-206.

17. Langlais PJ, Zhang SX. Cortical and subcortical white matter damage without Wernicke's encephalopathy after recovery from thiamine deficiency in the rat. Alcohol Clin Exp Res. 1997;21: 434-443.

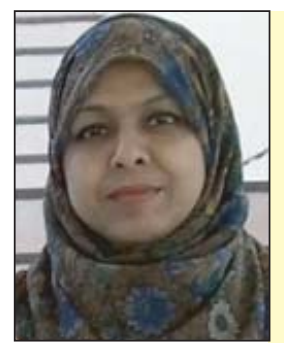

Professor Mahmuda Hassan passed MBBS, from Chittagong medical College, Chittagong in 1990. Completed MD (Paediatrics) from Bangladesh Institute of Child Health under the University of Dhaka in 1998. Obtained an advanced training on neonatal intensive care in 2007 from Birmingham Heartlands Hospital, Birmingham, UK. She has been teaching paediatrics for both undergraduate and postgraduate medical students at Ad-din Women's medical college. Contributed significantly to research relating to child health. She has around 50 publications in different reputed national and international medical journals. Author of a book written in Bengoli, Shishur Jonno Maer Dhud. Master trainer of different Paediatrics trainings at the national level under the government of Bangladesh. Now serving in Ad-din Women's Medical College in the field of paediatris. 